\title{
Opening Remarks
}

\author{
Shigeaki Hinohara, M.D. and Nobutani Doba, M.D*
}

Since Levine recommended the use of an arm chair in the relatively early phase of acute myocardial infarction (AMI), early ambulation and early discharge from the hospital have been widely accepted in the United States and other European countries and have resulted in a greatly improved rate of returning to work within a relatively short period! It has already been proved that a one-week hospital stay did not differ from a two-week stay in regard to morbidity and mortality from coronary heart disease (CHD) within a year after AMI? Seventy years ago, Herrick recommended to keep patients with AMI in beds as long as 6 weeks to avoid ventricular rupture and this concept was also supported by histological studies by Mallory and White 30 years later. In our country, these traditional concepts in management of AMI are still alive among many physicians involved in the care of these patients. Indeed, the symposium "Cardiac Rehabilitation" was held primarily for the education of those physicians who are not familiar with recent advances in the field of exercise cardiology, especially related to the management of patients with CHD. Eight out of 19 papers, which were initially submitted to us, were selected for presentation at this symposium and 2 additional papers were requested for special presentation in order to introduce the audience to the current trends of cardiac rehabilitation in Japan and throughout the world.

First Dr. Sada demonstrated natural changes in physical fitness after AMI. Influences of the severity of cardiac impairment on physical fitness levels seemed to appear in the first 6-month

\footnotetext{
Key Words:

Cardiac rehabilitation

Early mobilization

Exercise training

Acute myocardial infarction

A-C bypass surgery
}

period. Thereafter, physical fitness showed a gradual improvement for 2 to 3 years and was subsequently followed by an age-dependent natural decrease. Therefore, in the early posthospital phase it is mandatory to identify those patients who can participate in a physical training program. Dr. Saito described a 3-week program for in-hospital rehabilitation, of which feasibility and safety were clearly demonstrated with regard to improvement of physical fitness and of the rate of returning to work. He also pointed out a significantly higher incidence of cardiac events in the group with an ST depression than in the group with an ST elevation or without ST deviations. This fact seems to suggest that patients who show an ST depression during inhospital activity may have multi-vessel disease and a poor prognosis. Dr. Fujita showed a 4-week in-hospital program that was a shortened version of the previous 8-week program. The inhospital program of this type is common in many hospitals in Japan and is characterized by a reconditioning stage during the initial 2 weeks and by an in-hospital physical training stage during the subsequent 2 weeks. Since patients can walk in the corridors and take showers by the 14th hospital day in this program, those who can reach this stage without difficulties can be discharged from the hospital and may be able to increase their physical fitness by themselves according to a carefully instructed home exercise training program.

Dr. Sotobata developed 2 in-hospital exercise training protocols using a treadmill and steps which can be interchangeably applied to each other on the basis of an equidose concept expressed in terms of $\mathrm{VO}_{2}$. Dr. T.Takeuchi trained patients with stable effort angina and showed some results on parameters predicting the effectiveness of training. Psychiatric problems in AMI have been little studied in our country. Dr.

St. Luke's Nursing College; *The Life Planning Center, Tokyo, Japan 
Kurosawa stressed the importance of the careful assessment and management of these problems, especially in CCU. Although general needs for psychiatric consultations after AMI have been rare at least at the present time, this field seems to have been only recently opened for further detailed study in our country.

Dr. Inagaki compared 2 groups: one included patients who were managed according to a care program after discharge, including diet, exercise and other health educational instructions, and the other without this program. Cardiac events for 3 years after AMI seemed to be less in the former group associated with a higher rate of returning to work and with less anxiety or depression than in the latter group. At the Life Planning Center, the program for training patients with CHD has extensively been studied with special regard to continuous care with a team approach. This semi-supervised training program has been proven to be safe and effective, and this study emphasized the necessity of physical training of patients with poor physical fitness after discharge in order to differentiate their physical conditions: whether it is due to inadequate physical training or to the severity of underlying CHD. Dr. M.Takeuchi summarized the current trends of cardiac rehabilitation in Japan based on data collected from 20 hospitals and institutions belonging to the Council for Cardiac Rehabilitation Research. His data clearly demonstrated favorable effects of cardiac rehabilitation program on morbidity and mortality after AMI, although randomized trials on exercise training after AMI performed in other countries have never revealed such remarkable effects on longevity. ${ }^{3-5}$ Multifactorial interventions after AMI with special emphasis on optimum medical control and health education may contribute to a reduction of sudden deaths. 6 Dr. Toshima introduced modern trends and general concepts in cardiac rehabilitation in Western countries and some topics related to early mobilization, longterm effects of cardiac rehabilitation and effects of drug intervention, which were discussed at the 2nd World Congress of Cardiac Rehabilitation held in Jerusalem in 1981.

There are several reasons why social needs and physicians' interests concerning cardiac rehabilitation are still low in our country: 1) the incidence and prevalence of AMI have been far less than in other Western countries, 2) the health insurance system in our country has permitted patients to stay in a hospital for a relatively longer period and 3) patients with AMI are relatively older in Japan than in other countries. Identification of patients for early mobilization and early discharge is thought to be mandatory at the present time. All patients without complications should be involved in a post-hospital home rehabilitation program after discharge with medical, psychological and social precautions. If patients cannot improve their physical fitness levels in the phase 2 of cardiac rehabilitation, whether it is due to inadequate exercise training or the severity of underlying CHD should be clarified. Essentially the same rehabilitation program as in AMI can also be applied to patients after aorto-coronary bypass surgery.

Further studies and many more experiences with early mobilization and early discharge, patient selection for home exercise training and proper physical training in the 2 nd phase and identification of high risk patients through these procedures are necessary in order to develop a standardized program for the proper management of patients with AMI.

\section{REFERENCES}

1. WENGER NK, HELLERSTEIN HK, BLACKBURN H, CASTRANOVA SJ: Physician practice in the management of patients with uncomplicated myocardial infarction: Changes in the past decade. Circulation 65: 421, 1982

2. McNEER JF, WAGNER GS, GINSBURG PB, WALLACE AG, MCCANTS CV, CONLEY MJ, ROSATI RA: Hospital discharge after acute myocardial infarction. $N$ Engl J Med 298: 229, 1978

3. KENTALA E: Physical fitness and feasibility of physical rehabilitation after myocardial infarction in men of working age. Ann Clin Res 4 (Suppl 9): 1,1972

4. WILHELMSEN L, SANNE H, ELMFELD D, GRIMBY G, TIBBLIN G, WEPEL H: A controlled trail of physical training after myocardial infarction: Effects on risk factors, nonfatal reinfarction, and death. Prevent Med 4: 491, 1975

5. The National Exercise and Heart Disease Project: Effects of a prescribed supervised exercise program on mortality and cardiovascular morbidity in patients after a myocardial infarction. $A m J$ Cardiol 48: 39, 1981

6. KALLIO V, HAMALAINEN H, HAKKILA J, LUURILA O: Reduction in sudden deaths by a multifactorial intervention program after acute myocardial infarction. Lancet ii: 109, 1979 